Cite this: Phys. Chem. Chem. Phys., 2013 15, 7147

Received 20th December 2012, Accepted 1st March 2013

DOI: $10.1039 / \mathrm{c} 3 \mathrm{cp} 44611 \mathrm{e}$

www.rsc.org/pccp

\section{Interpretation of experimental hydrogen-bond enthalpies and entropies from COSMO polarisation charge densities $\uparrow$}

\author{
Andreas Klamt, ${ }^{\text {ab }}$ Jens Reinisch, ${ }^{a}$ Frank Eckert, ${ }^{a}$ Jérôme Graton ${ }^{c}$ and \\ Jean-Yves Le Questel*c
}

\begin{abstract}
In this work, experimental hydrogen-bond (HB) enthalpies measured in previous works for a wide range of acceptor molecules in dilute mixtures of 4-fluorophenol in non-polar solvents are quantified from COSMO polarisation charge densities $\sigma$ of HB acceptors (HBA). As well as previously demonstrated for quantum chemically calculated $\mathrm{HB}$ enthalpies, a good correlation of the experimental data with the polarisation charge densities is observed, covering an extended range of $\mathrm{HBA}(\mathrm{O}, \mathrm{N}, \mathrm{S}, \pi$ systems and halogens) ranging from very weak to strong hydrogen bonds. Furthermore, for the first time, a quantitative analysis of experimental HB entropies is performed for such a chemical diversity of HBA. A good quantification of these entropies is achieved using the polarisation charge density $\sigma$ as a descriptor in combination with the logarithm of a directional partition function $\Omega_{\mathrm{HB}}$. This partition function covers the directional and multiplicity entropy of HBA and is based on the $\sigma$-proportional HB enthalpy expression taken from COSMO-RS. As a result, the experimental $\mathrm{HB}$ enthalpies and free energies of the $\sim 300 \mathrm{HB}$ complexes are quantified with an accuracy of $\sim 2 \mathrm{~kJ} \mathrm{~mol}^{-1}$ based on COSMO polarisation charge densities.
\end{abstract}

\section{Introduction}

The hydrogen bond (HB) is recognised as the most important specific interaction between a molecule and its local environment. However, there is a tendency to view hydrogen-bond acceptors (HBA) and donors (HBD) as atomic sites and to consider them equivalent despite that the effects of organic functions and substituents that define the local molecular environment can have huge impacts on their HB properties. Indeed, although HBs are qualitatively well understood, it is generally admitted that quantitative data are needed. From an experimental point of view, the quantification of HB energy is complicated because a hydrogen bond can never be studied individually,

\footnotetext{
${ }^{a}$ COSMOlogic GmbH\&CoKG, Burscheider Str. 515, 51381 Leverkusen, Germany.

E-mail: klamt@cosmologic.de; Tel: +492171 731681

${ }^{b}$ Institute of Physical and Theoretical Chemistry, University of Regensburg, Germany

${ }^{c}$ Chimie et Interdisciplinarité: Synthèse, Analyse, Modélisation (CEISAM), UMR CNRS 6230, Université de Nantes, 2 rue de la Houssinière - BP 92208, 44322 Nantes Cedex 3, France. E-mail: jean-yves.le-questel@univ-nantes.fr; Tel: +33251125563

† Electronic supplementary information (ESI) available. See DOI: 10.1039/ c3ср44611e
}

its formation going unavoidably along with the concomitant dispersion and electrostatic contributions.

The situation is even worse in solution, since the formation of a hydrogen bond in this case additionally requires the removal of the solvent molecules in interaction with the acceptor prior to the HB formation. If the solvent molecules are not completely isotropic with respect to their electrostatics, or if they even have their own polar hydrogen atoms, i.e. their own prospective HBD site, the estimation of the free energy needed for removing the solvent at the position of the acceptor (desolvation) even requires statistical thermodynamics for getting appropriate averages.

The importance of hydrogen-bonding and the need of quantitative parameters for a comprehensive understanding and description of the interaction has led several research groups to concentrate their efforts in the construction of $\mathrm{HB}$ basicity scales, called $\mathrm{p} K_{\mathrm{HB}},{ }^{1} \log K_{\beta},{ }^{2} \Sigma \beta_{2}{ }^{\mathrm{H}},{ }^{3,4} \Sigma C_{a}{ }^{5,6}$ or $B{ }^{7}$ Following the pioneering work of Taft and coworkers, ${ }^{1}$ Laurence et $a l^{8}$ reported recently the development of the $\mathrm{p} K_{\mathrm{BHX}}$ database, which contains an unprecedented set of experimental values related to $\mathrm{HB}$ basicity. More precisely, $\mathrm{HB}$ free energies have been determined in tetrachloromethane $\left(\mathrm{CCl}_{4}\right)$ for a large number of chemically diverse HBA molecules using $p$-fluorophenol $(p \mathrm{FP})$ as 
the reference HBD, chosen for historical and technical reasons., ${ }^{1,9}$ The measurements are made following the standard procedure based on the absorbance decrease in the $\mathrm{OH}$ absorption of $p \mathrm{FP}$ $\left(\nu(\mathrm{OH})=3614 \mathrm{~cm}^{-1} ; \varepsilon=237 \mathrm{dm}^{3} \mathrm{~mol}^{-1} \mathrm{~cm}^{-1}\right)$ in dilute ternary $p$ FP-base- $\mathrm{CCl}_{4}$ solutions at $25{ }^{\circ} \mathrm{C}$, leading to $\mathrm{p} K_{\mathrm{BHX}}$ values. More precisely, typical concentrations of 3-4 $\mathrm{mM}$ are prepared for our reference donor $(p \mathrm{FP})$ and of $10-300 \mathrm{mM}$ for the acceptors, depending on their HB basicity. Although contaminated to some degree with local desolvation and electrostatic contributions, the HB free energy is estimated from the equilibrium constants of complex association as $-\mathrm{RT} \ln K$, or equivalently as $-\mathrm{RT}$ $\mathrm{p} K_{\mathrm{BHX}} / \ln 10$. Using this notation, a strong $\mathrm{HB}$ acceptor forming a hydrogen-bonded complex with a large association constant $(K)$, that is a low dissociation constant $\left(K_{\mathrm{BHX}}=1 / K\right)$, has a large positive value of $\mathrm{p} K_{\mathrm{BHX}}$. This can be reasonably justified based on the aprotic nature of $\mathrm{CCl}_{4}$, its high degree of isotropy with respect to polarity, and the use of a reference HBD. In its present version, the $\mathrm{p} K_{\mathrm{BHX}}$ database contains about 1340 values corresponding to nearly $1200 \mathrm{HBA}$. Furthermore, the HB enthalpies towards $p$ FP for about 310 varied organic bases, ranging over $40 \mathrm{~kJ} \mathrm{~mol}^{-1}$, have been published, ${ }^{10}$ providing, in addition to the HB free energies, the quantitative establishment of the order of $\mathrm{HB}$ enthalpies according to a given atomic acceptor site. More exactly, this order of HB enthalpies has even been established according to the chemical function to which belongs the studied atomic site. These values have been measured from the temperature dependence determinations of the complex equilibrium constants. For this energetically extended and chemically diversified set of acceptors, the HB free energy was split into its enthalpic and entropic contributions. To our knowledge, this work is therefore the most extensive homogeneous experimental study of the thermodynamics of hydrogen bonding that has been carried out so far.

From a theoretical perspective various attempts have been presented aiming for a predictive quantification of hydrogenbond strength. Most often the HBD and HBA strength parameters used as experimental basis are Abraham's ones. ${ }^{2-4}$ Beyond more empirical increment schemes ${ }^{11}$ quantum chemical descriptors have been used for a correlation of HB strength. Kenny ${ }^{12}$ used the electrostatic potential as a quantum chemical descriptor for HBA strength of nitrogen bases. Schwöbel and coworkers ${ }^{13-16}$ presented several studies, in which they demonstrate the suitability of local frontier orbital descriptors ${ }^{17}$ for the predictive quantification of Abraham's HBA and HBD parameters of a chemically much broader range of donor and acceptor sites.

Klamt et $a .^{18}$ recently presented a detailed study on $\mathrm{HB}$ enthalpies calculated quantum chemically for 2465 donor acceptor pairs. For reflecting the situation in a solvent, solvation effects were considered in the extreme limit of a perfect conductor employing the COSMO continuum solvation model. ${ }^{19}$ On a very large and chemically very diverse set of $\sim 2500$ donoracceptor complexes, they demonstrated that the quantum chemically calculated HB enthalpies can be quantified with an accuracy of $\sim 1.3 \mathrm{~kJ} \mathrm{~mol}^{-1}$ assuming a bilinear dependence on the COSMO polarisation charge densities $\sigma_{\text {don }}$ and $\sigma_{\text {acc }}$. The COSMO polarisation charge density is the local surface density of the conductor screening charges, i.e. the screening charge per unit area of the cavity surface, as resulting from the conductorboundary conditions in the continuum solvation model (COSMO). The suitability of the polarisation charge density for the quantification of intermolecular interaction has been demonstrated by Klamt in his COSMO-RS solvation model. ${ }^{20-22}$ For those not familiar with the concept of COSMO, a more detailed description and discussion of the polarisation charge density $\sigma$ is given in appendix SI1 in the ESI. $\uparrow$ The study on the quantum chemically calculated $\mathrm{HB}$ complexes confirmed the bilinear $\sigma$-dependence of HB bond energies assumed within the COSMO-RS.

In this paper we demonstrate that the described linearity of $\mathrm{HB}$ energies with respect to $\sigma_{\text {acc }}$ does not only hold for the calculated $\mathrm{HB}$ energies, but also for the experimental $\mathrm{HB}$ enthalpies reported by Laurence et $a l^{10}$ An analysis of the $\mathrm{HB}$ free energy with respect to $\sigma_{\text {acc }}$ clearly discloses that the directional $\mathrm{HB}$ entropy has an important influence. Furthermore, we introduce a directional HB partition function which allows for the quantification of this directional entropy without any additional adjusted parameter.

\section{Data sets and calculation methods}

For the sake of consistency, we will only consider in this paper the 309 HBA molecules for which HB enthalpies $\Delta H_{\mathrm{HB}}^{\mathrm{o}}$ and $\mathrm{HB}$ free energies $\Delta G_{\mathrm{HB}}^{\mathrm{O}}$ with $p \mathrm{FP}$ in $\mathrm{CCl}_{4}$ or tetrachloroethylene $\left(\mathrm{C}_{2} \mathrm{Cl}_{4}\right)$ have been reported by Laurence et al. ${ }^{10}$ in the core and in the supplementary data of their paper. Some compounds (e.g. amines) are known to react with $\mathrm{CCl}_{4}$, and the corresponding measurements were therefore made in $\mathrm{C}_{2} \mathrm{Cl}_{4}$, which is very similar to $\mathrm{CCl}_{4}$, in particular with respect to polarity. The differences between the HB data obtained in the two solvents have indeed been shown to be small and no systematic differences were observed. ${ }^{23}$

For all compounds, DFT/COSMO calculations have been performed with the TURBOMOLE program $^{24}$ at the BP-TZVPD/ COSMO level, ${ }^{25-30}$ as described in ref. 18. A conformational analysis following the concepts of COSMOconf workflow ${ }^{31}$ was performed for each of the acceptor molecules. For each atom the maximum of the locally averaged polarisation charge density $\sigma$ was calculated with the COSMOtherm program ${ }^{32}$ according to eqn (11) of ref. 21. Since negatively polar HBAs need to have positive $\sigma$-values, the atom with the maximum $\sigma$-value, i.e. the most polar acceptor, was selected as the HBA of interest in the case of polyfunctional molecules, bearing several HBAs. Actually, these theoretical assignments disagreed from those assumed in the experimental work only in one case, which will be discussed below.

While in the previous study on the $\sigma$-dependence of QM/COSMO calculated HB enthalpies the molecular conformation was known for each donor and acceptor under consideration, the comparison with experimental HB enthalpies and free energies bears the complication that for some of the more flexible acceptors, the exact or predominant conformation in the solvent is not known from experiment. The most relevant conformation for hydrogen bonding may even be, in some cases, 


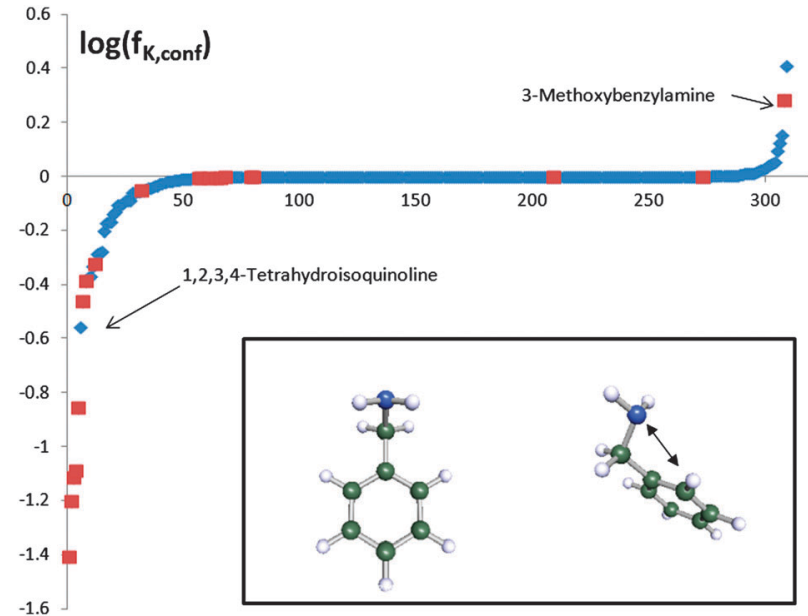

Fig. 1 Conformational sensitivity of the calculated complex formation constant. The 309 compounds are ordered with respect to the ratio $f_{k, c o n f}$ of the donoracceptor contact probability calculated without and with conformational multiplicity. The red symbols mark the compounds removed from the sample (see the text). The inset illustrates the preferred conformation of benzylamine in polar solvents with an exposed nitrogen lone-pair, and the preferred conformation in a non-polar solvent as $\mathrm{CCl}_{4}$, in which the nitrogen lone-pair interacts with an orthohydrogen atom and thus is less available for hydrogen bonding.

different from the predominant conformation in the solvent. To appropriately deal with such conformational ambiguities, we carried out a COSMO-RS study of the complex formation constants by calculating the contact probability of the dilute acceptor molecules and dilute $p \mathrm{FP}$ molecules in $\mathrm{CCl}_{4}$ with the COSMOtherm program. ${ }^{32}$ At first, that calculation was performed for the entire conformational set, yielding, aside of the conformationally averaged contact probability, the population of the individual conformations in $\mathrm{CCl}_{4}$. Then we repeated the calculation of the complex formation constant only taking into account the most populated conformation in $\mathrm{CCl}_{4}$. The ratio $f_{\mathrm{K}, \text { conf }}$ of the calculated complex formation constants without and with conformational multiplicity was calculated and is reported in Table SI1 in the ESI. $†$ As shown in Fig. 1, for most of the considered acceptors the complex formation constant is rather insensitive to the conformational multiplicity. Only for seven compounds we found $\log \left(f_{\mathrm{K}, \mathrm{conf}}\right)$ to be lower than -0.4, all of them being benzylamines, except 1,2,3,4-tetrahydroisoquinoline, the extreme value being -3.2 log-units for 3-(trifluoromethyl)benzylamine. For these molecules, our calculations show that the lowest energy conformation in $\mathrm{CCl}_{4}$ is much less involved in HB interactions than the other conformations. A closer investigation showed that this behaviour is due to the formation of a 5-membered ring stabilized by a weak intramolecular interaction of the slightly positive ortho-hydrogen with the amine lone pair, shown in the right inset in Fig. 1. On that basis, the 18 amines having the same 5-membered ring structure in their lowest energy conformer in $\mathrm{CCl}_{4}$ have been removed from our sample. Being a single exception, we kept 1,2,3,4-tetrahydroisoquinoline in the data set. For all compounds with $\left|\log \left(f_{\mathrm{K}, \mathrm{conf}}\right)\right|$ less than 0.4 , we estimate that the potential error resulting from the neglect of additional conformations on the free energies of $\mathrm{HB}$ formation should be smaller than $\sim 2 \mathrm{~kJ} \mathrm{~mol}^{-1}$.

For two very bulky tertiary amine compounds ( $N, N$-diisopropyl-3-pentylamine and $N, N$-diisopropylisobutylamine) a more serious problem occurred. In all conformations generated by COSMOconf the amine lone-pair was entirely hidden by the bulky substituents. No surface area with sufficiently high $\sigma$ for hydrogen bonding was found. Even manual attempts to generate the conformations with the accessible amine lonepair failed, since the quantum chemical energies of these conformations were too high in order to be relevant. These theoretical trends agree well with the experimental behaviour observed for tertiary amines ${ }^{33}$ for which the nitrogen is hidden by long and/or branched alkyl chains. Consistently, these two compounds have also been removed from our analysis dataset, which finally consisted of 289 acceptor molecules.

\section{Results and discussion}

As the first step of our analysis, we plotted the experimental HB enthalpy $\Delta H_{\mathrm{HB}}^{\mathrm{o}} v s$. the polarisation charge density $\sigma_{\text {acc }}$ of the strongest acceptor position, as shown in Fig. 2. A linear dependence can clearly be observed. This relationship is characterised by a correlation coefficient $r^{2}$ of 0.935 , and a standard deviation of $2.4 \mathrm{~kJ} \mathrm{~mol}^{-1}$ with a rather homogeneous error distribution. Hence, we may conclude as a first result that the $\mathrm{HB}$ enthalpies measured in $\mathrm{CCl}_{4}$ confirm the assumption of $\sigma$-proportionality of the $\mathrm{HB}$ enthalpy as suggested in our previous theoretical study. ${ }^{18}$

Next, we considered the analogous plot for the experimental HB free energy $\Delta G_{\mathrm{HB}}^{\mathrm{o}}$, as shown in Fig. 3. The $\Delta G_{\mathrm{HB}}^{\mathrm{o}}$ data are the primary results of the equilibrium constant determination while the enthalpies are derived from the van't Hoff plot established with the equilibrium constants measured on a range of $60{ }^{\circ} \mathrm{C}$, from $-5{ }^{\circ} \mathrm{C}$ to $55{ }^{\circ} \mathrm{C}$. For this reason, the experimental error is found to be smaller for $\Delta G_{\mathrm{HB}}^{\mathrm{o}}\left( \pm 0.2 \mathrm{~kJ} \mathrm{~mol}^{-1}\right)$ than for $\Delta H_{\mathrm{HB}}^{\mathrm{o}}\left( \pm 1 \mathrm{~kJ} \mathrm{~mol}^{-1}\right)$. However, despite the higher experimental accuracy for $\Delta G_{\mathrm{HB}}^{\mathrm{o}}$, the correlation with the polarisation charge density $\sigma_{\text {acc }}$, with $r^{2}=0.729$ and $s=3.0 \mathrm{~kJ} \mathrm{~mol}^{-1}$, is much worse than the corresponding one found for $\Delta H_{\mathrm{HB}}^{\mathrm{o}}$. This is not really unexpected since the entropic contribution arising from site and lone-pair multiplicity, from the degree of directional softness and from the vibrational changes arising from the deep and narrow potential energy minima typical for HB cannot be assumed to be simply proportional to $\sigma_{\text {acc }}$. After a closer analysis of the deviations from the regression line, it turns out that the $\sigma$-regression strongly underestimates $\Delta G_{\mathrm{HB}}^{\mathrm{o}}$ for exposed oxygen atoms, the extreme case corresponding to hexamethylphosphoramide, while on the upper side of the regression line the $\Delta G_{\mathrm{HB}}^{\mathrm{o}}$ obtained are in contrast overestimated for sterically hindered acceptor atoms such as tertiary amine nitrogens. This observation highlights the need of a quantitative measure of the directional entropy, i.e. of the number of almost equivalent positions on the molecular surface at which the hydrogen bond can be formed, in the HB free energy.

The entropic contribution arising from the multiplicity is to some extent already taken into account in the experimental data, 


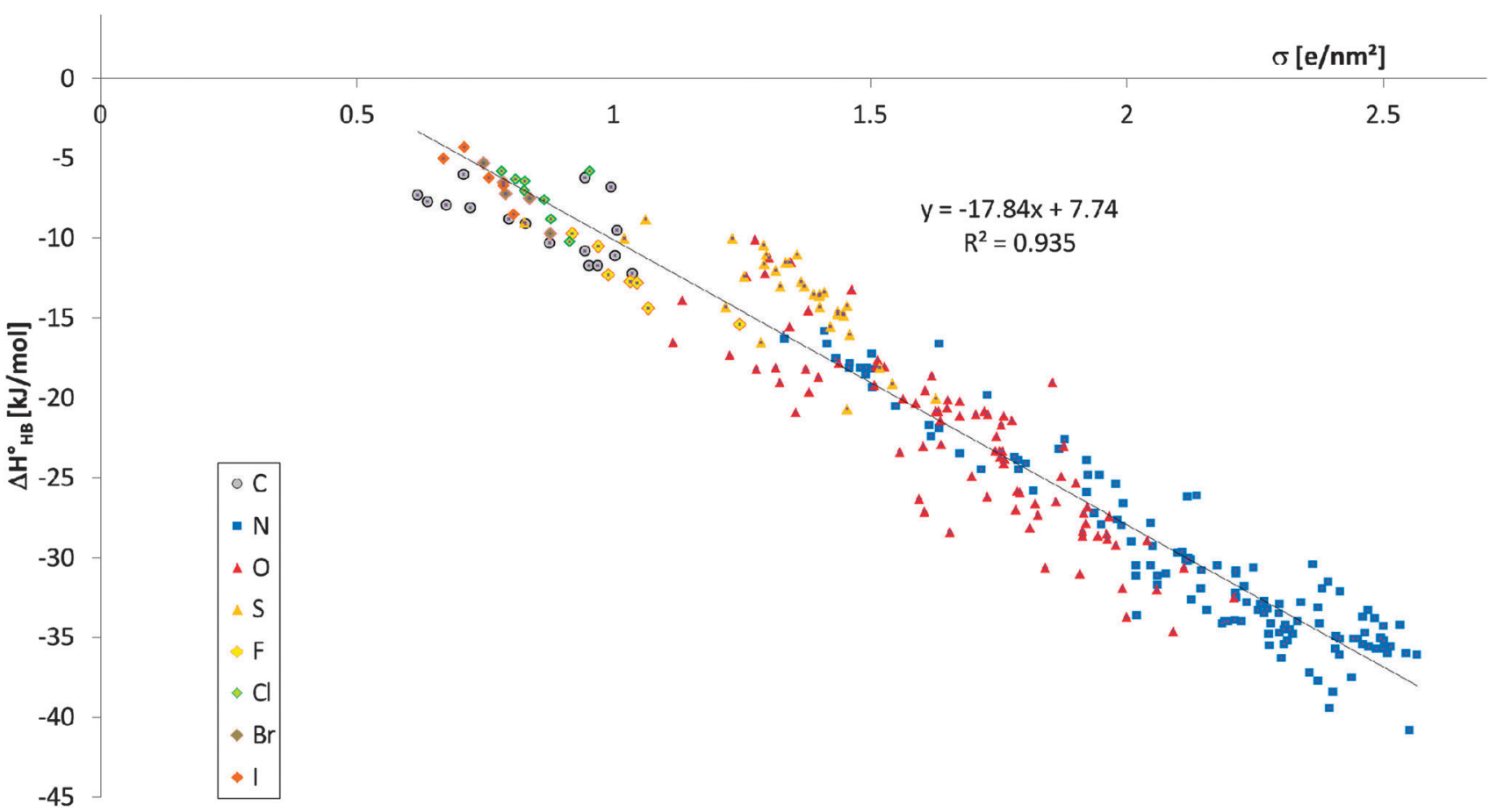

Fig. 2 Experimental HB enthalpies, $\Delta H_{\mathrm{HB}}^{\circ}$, of 289 acceptor molecules plotted vs. the COSMO polarisation charge density $\sigma_{\text {acc }}$ of the most polar acceptor position.

since the entropic values $-\Delta S^{0}$ are statistically corrected by a $-R \ln (n)$ term, where $n$ is the number of (quasi)-equivalent sites, in order to put these values on a per acceptor atom basis. However, some cases have been trickier to handle. Thus, the aromatic bases have been treated considering one benzene ring as a single HBA. In the same vein, for molecular systems bearing two HBAs in close spatial proximity (e.g. syn-2,4-difluoroadamantane) the experimental values refer to one HBA site, based on the experimental and theoretical evidence of bifurcated (three-centre) HB interactions in these cases. ${ }^{34}$ Lastly, in the case of polyfunctional bases, experimental arguments have been used as guides for the statistical correction to apply. For example,

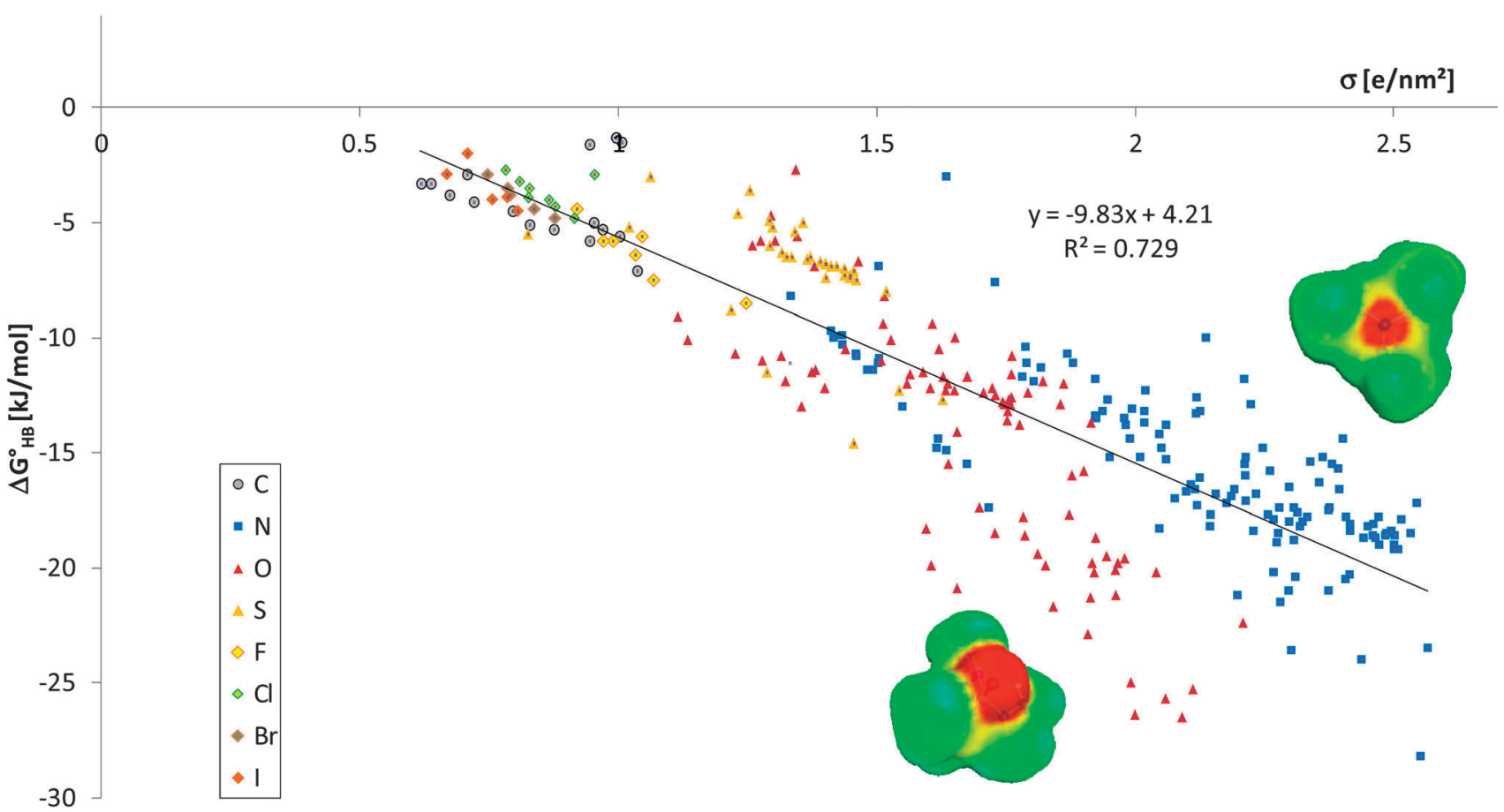

Fig. 3 Experimental $\mathrm{HB}$ free energies, $\Delta \mathrm{G}_{\mathrm{HB}}^{\circ}$, of 289 acceptor molecules plotted vs. the COSMO polarisation charge density $\sigma_{\text {acc }}$ of the most polar acceptor position. 
in the case of triethylthiophosphate, which possesses three oxygen and one sulphur atoms as potential HBAs, we have considered the sulphur atom as the only HBA site, based on a previous experimental investigation. ${ }^{35}$ This last assignment is in contradiction with the trends obtained from the computed polarisation charge densities $\sigma_{\text {acc }}$, since the three oxygen atoms are found to be the strongest HBA sites.

These various situations have led us to the decision to return to the unbiased original $\Delta G^{\mathrm{o}}$ values for the entire sample of molecules, and to calculate the full $\mathrm{HB}$ directional and multiplicity entropic correction by a HB partition function, evaluated over the entire molecular surface. Based on the assumption of $\sigma$-proportional HB enthalpy, and using the slope of the regression line in Fig. 1 as a reasonable guess for the dependence of the local $\mathrm{HB}$ enthalpy on $\sigma_{\text {acc }}$, we thus constructed a $\mathrm{HB}$ partition function $\Omega_{\mathrm{HB}}$ and the respective entropy descriptor $S_{\mathrm{HB} \text { dirmult }}=R \ln \left(\Omega_{\mathrm{HB}}\right)$, which is expected to capture this missing entropic contributions. For details of the calculation of $\Omega_{\mathrm{HB}}$ see Appendix 1. As a side product, this partition function also gives us an expectation value $\left\langle\sigma_{\text {acc }}\right\rangle$ which should be a better measure for the enthalpy than the maximum value.

As can be seen in Fig. 4, the correlation between the experimentally reported $\Delta G_{\mathrm{HB}}^{\mathrm{o}}$ values, corrected by $-\mathrm{RT} \ln \left(\Omega_{\mathrm{HB}}\right)$, and $\sigma_{\text {acc }}$ is strongly improved $\left(r^{2}=0.923\right.$ and $\left.s=2.1 \mathrm{~kJ} \mathrm{~mol}^{-1}\right)$. This goes along with a significant change in the slope of the regression line. We attribute this to the fact that the directional entropy is to some degree correlated with $\sigma_{\text {acc. }}$. This is because the nitrogen acceptors, and especially the tertiary amines, have the highest values of $\sigma_{\text {acc}}$, but very small exposed surface area available for the single lone-pair, while oxygen acceptors typically have smaller values of $\sigma_{\text {acc }}$ and two lone-pairs, i.e. larger HB area. Finally, the very weak halogen and $\pi$-acceptors have small $\sigma_{\text {acc }}$ values but a large accessible surface for complexation of quite similar $\sigma$, i.e. a large directional entropy.

If we replace the maximum of the acceptor polarisation charge density, so far denoted $\sigma_{\text {acc }}$, by the expectation value $\left\langle\sigma_{\text {acc }}\right\rangle$, and replace the reference $\sigma$ in the partition function accordingly, then the statistics does not change, i.e. we still have $r^{2}=0.923$ and $s=2.1 \mathrm{~kJ} \mathrm{~mol}^{-1}$. But if we replace the descriptor $\sigma_{\text {acc }}$ by $\left\langle\sigma_{\text {acc }}\right\rangle$ in the enthalpy correlation, i.e. in an analogue of Fig. 1, the correlation improves to $r^{2}=0.945$ and $s=2.2 \mathrm{~kJ} \mathrm{~mol}^{-1}$, while the slope of the regression is almost unaffected. Hence, overall, the hydrogen-bond expectation value $\left\langle\sigma_{\text {acc }}\right\rangle$ seems to be the slightly better descriptor, but from a practical perspective the local maximum value $\sigma_{\text {acc }}$ is more readily available.

Going a next step toward thermodynamic consistency, we can calculate the partition functions and expectation values taking into account multiple conformations of the acceptor. This requires the knowledge of the conformational population $w_{i}$ of each conformation $i$. If we use the COSMO-RS model in its COSMOtherm implementation, we get estimates of these conformational populations in the solvent. Based on these we can easily get the respective $\mathrm{HB}$ partition functions $\Omega_{\mathrm{HB} \text {,conf }}$ and expectation values $\left\langle\sigma_{\text {acc }}\right\rangle_{\text {conf }}$. Inserting these values we end up with a tiny improvement for the HB enthalpy regression $\left(r^{2}=\right.$ 0.946, $s=2.2 \mathrm{~kJ} \mathrm{~mol}^{-1}$; see Fig. 4) and also for the HB free energy regression $\left(r^{2}=0.924, s=2.1 \mathrm{~kJ} \mathrm{~mol}^{-1}\right.$; see Fig. 5). Since we had already excluded the 18 compounds showing conformational preferences with specific intramolecular interactions,

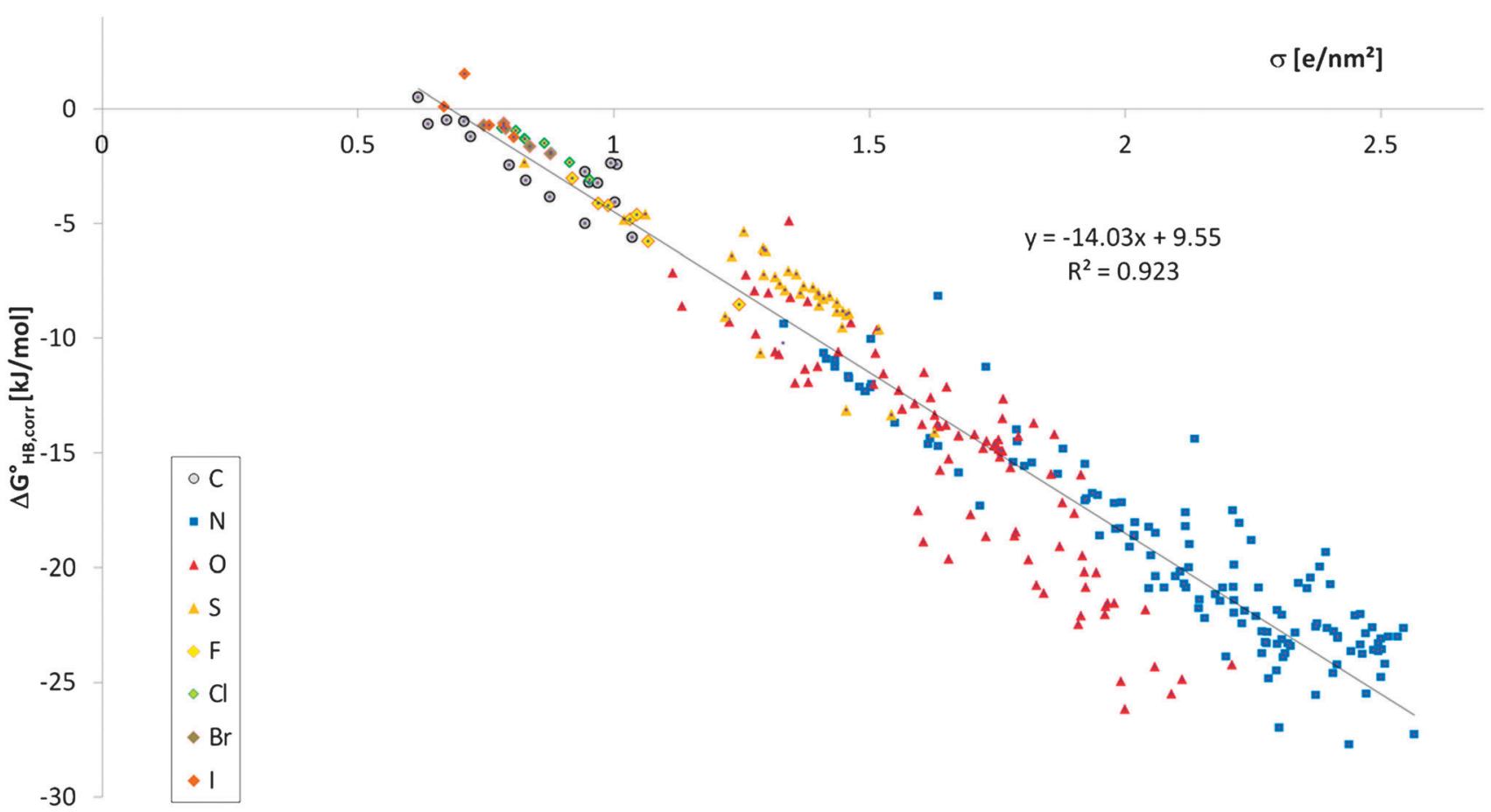

Fig. $4 \mathrm{HB}$ free energies, $\Delta \mathrm{G}_{\mathrm{HB}, \text { corr }}^{\circ}$ corrected from the directional and multiplicity entropy contributions of 289 acceptor molecules plotted vs. the COSMO polarisation charge density $\sigma_{\text {acc. }}$. 


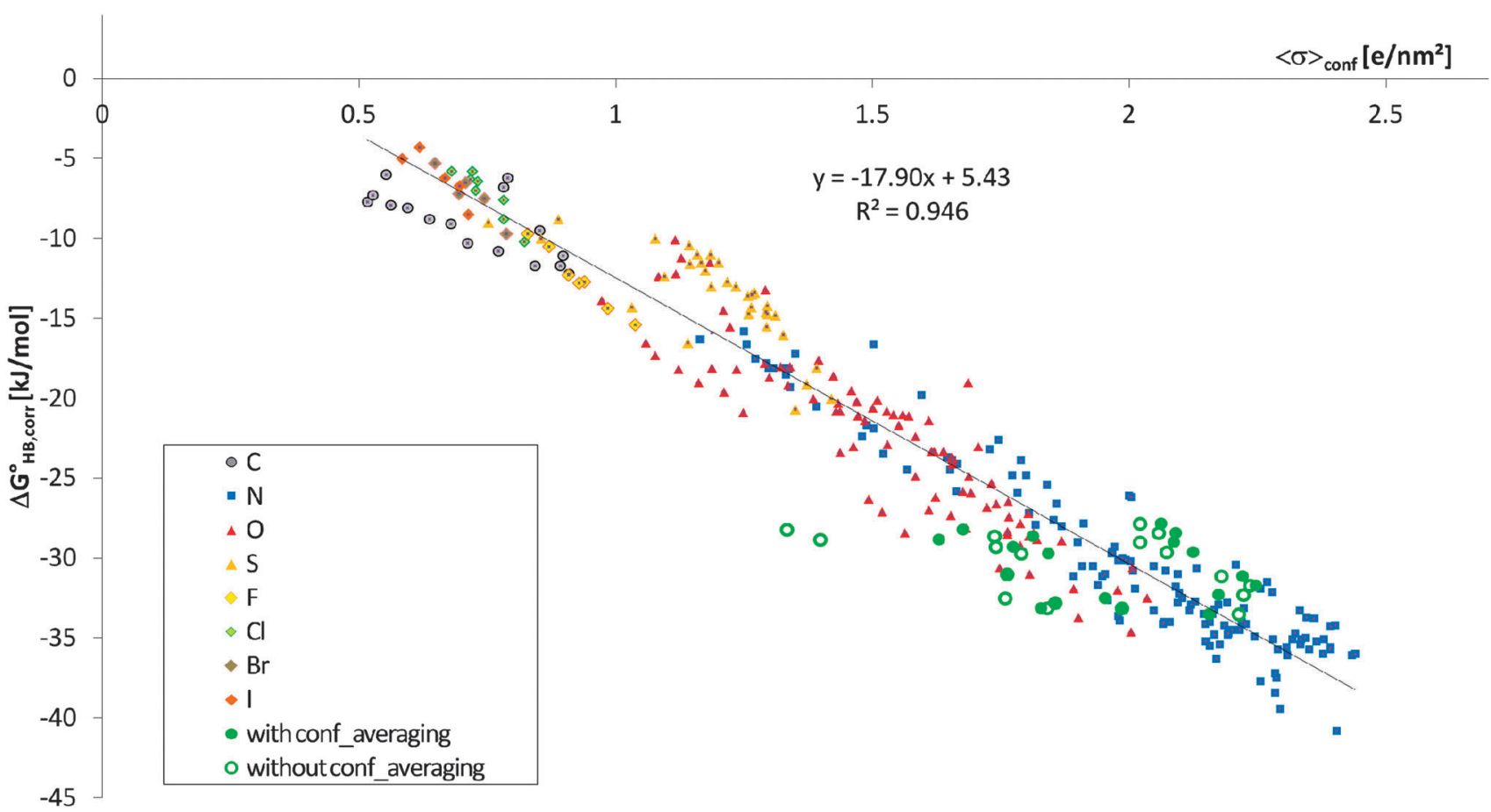

Fig. 5 Experimental $\mathrm{HB}$ enthalpies, $\Delta H_{\mathrm{HB}}^{\circ}$, of the $307 \mathrm{HBA}$ molecules $(289+18)$ plotted vs. the $\mathrm{HB}$ expectation value of the COSMO polarisation charge density $\left\langle\sigma_{\text {acc }}\right\rangle_{\text {conf }}$. The full green symbols mark the 18 conformational problematic cases (mainly benzylamines). For comparison, open green symbols mark these compounds before conformational averaging.

it is not surprising that the improvement in this dataset is small. But, as shown in Fig. 5 and 6, these 18 HBAs are now described with the same quality as the other compounds using the conformationally averaged quantities.

Based on the two regressions (in units of $\mathrm{e} \mathrm{nm}^{-2}$ for $\sigma$ and $\mathrm{kJ} \mathrm{mol}^{-1}$ for energy)

$$
\Delta H_{\mathrm{HB}}^{\mathrm{o}}=-17.9\left\langle\sigma_{\mathrm{acc}}\right\rangle+5.4
$$

and

$$
\Delta G_{\mathrm{HB}}^{\mathrm{o}}=-14.1\left\langle\sigma_{\mathrm{acc}}\right\rangle+10.1-\mathrm{RT} \ln \left(\Omega_{\mathrm{HB}}\right)
$$

we yield

$$
\begin{aligned}
\Delta S_{\mathrm{HB}}^{\mathrm{o}}=\left(\Delta H_{\mathrm{HB}}^{\mathrm{o}}-\Delta G_{\mathrm{HB}}^{\mathrm{o}}\right) / 298 \mathrm{~K}= & -3.8\left\langle\sigma_{\mathrm{acc}}\right\rangle / 298 \mathrm{~K}-4.7 / 298 \mathrm{~K} \\
& +R \ln \left(\Omega_{\mathrm{HB}}\right)
\end{aligned}
$$

Hence the HB entropy consists of an acceptor independent negative constant, a directional and multiplicity contribution and a major $\sigma$-proportional entropy loss. The latter can be easily interpreted by the deeper and thus more narrow minimum on the molecular potential energy surface with increasing $\mathrm{HB}$ enthalpy, and hence with increasing $\sigma_{\text {acc }}$.

It might be worth noting that the $\sigma$-slope of the HB enthalpies found here, i.e. the value of $-17.9 \mathrm{~kJ} \mathrm{~mol}^{-1} \mathrm{~nm}^{2} \mathrm{e}^{-1}$, is only $63 \%$ of the $\sigma$-slope of the regression line found in eqn (1) in the study on quantum chemically calculated HB enthalpies in a conductor environment ${ }^{18}$ for a donor of $\sigma_{\mathrm{don}}=2.0$, i.e. using the value found on the donor hydrogen atom of $p$ FP. Hence, in a conductor, the hydrogen bonds would be roughly $50 \%$ stronger than in $\mathrm{CCl}_{4}$. This is not surprising given the stronger polarisation of the donor and acceptor molecules in a conductor compared with the non-polar $\mathrm{CCl}_{4}$ environment used here. Indeed, this is in quite good agreement with the fact that the polarisation charge densities in a non-polar solvent typically are by a factor of 0.8 smaller than in a conductor, due to the almost complete lack of electronic back-polarisation in non-polar solvents. ${ }^{36}$ Since we are using as descriptors $\sigma$ values calculated in a conductor environment here, and since this reduction applies to the donor and acceptor, we would expect a reduction of the slope of $\Delta H_{\mathrm{HB}}^{\mathrm{o}}, v s . \sigma$ by a factor of $\sim 0.8^{2}=64 \%$.

\section{Summary and conclusions}

Experimental $\mathrm{HB}$ free energies and enthalpies measured in $\mathrm{CCl}_{4}$ for a wide and varied selection of HB bases have been analysed from COSMO polarisation charge densities $\sigma_{\text {acc }}$ of the acceptor atoms. The HB enthalpies show a strong correlation with $\sigma_{\text {acc }}$ with $r^{2}=0.94$ and a standard deviation of $\sim 2 \mathrm{~kJ} \mathrm{~mol}^{-1}$. Based on the observed $\sigma$-proportionality of the HB enthalpies, partition functions have been constructed for the molecular free energy of hydrogen bonding. Using these partition functions for the description of the directional and multiplicity entropy of hydrogen bonding, the HB free energy can as well be described as a linear function of $\sigma_{\text {acc }}$ with almost the same correlation coefficient and standard deviation. The lower slope of the HB free energy $\sigma$-regression compared to the HB enthalpy regression corresponds to a $\sigma$-proportional part of the HB entropy.

The relations presented herein between experimental $\mathrm{HB}$ enthalpies or free energies and the COSMO polarisation charge 


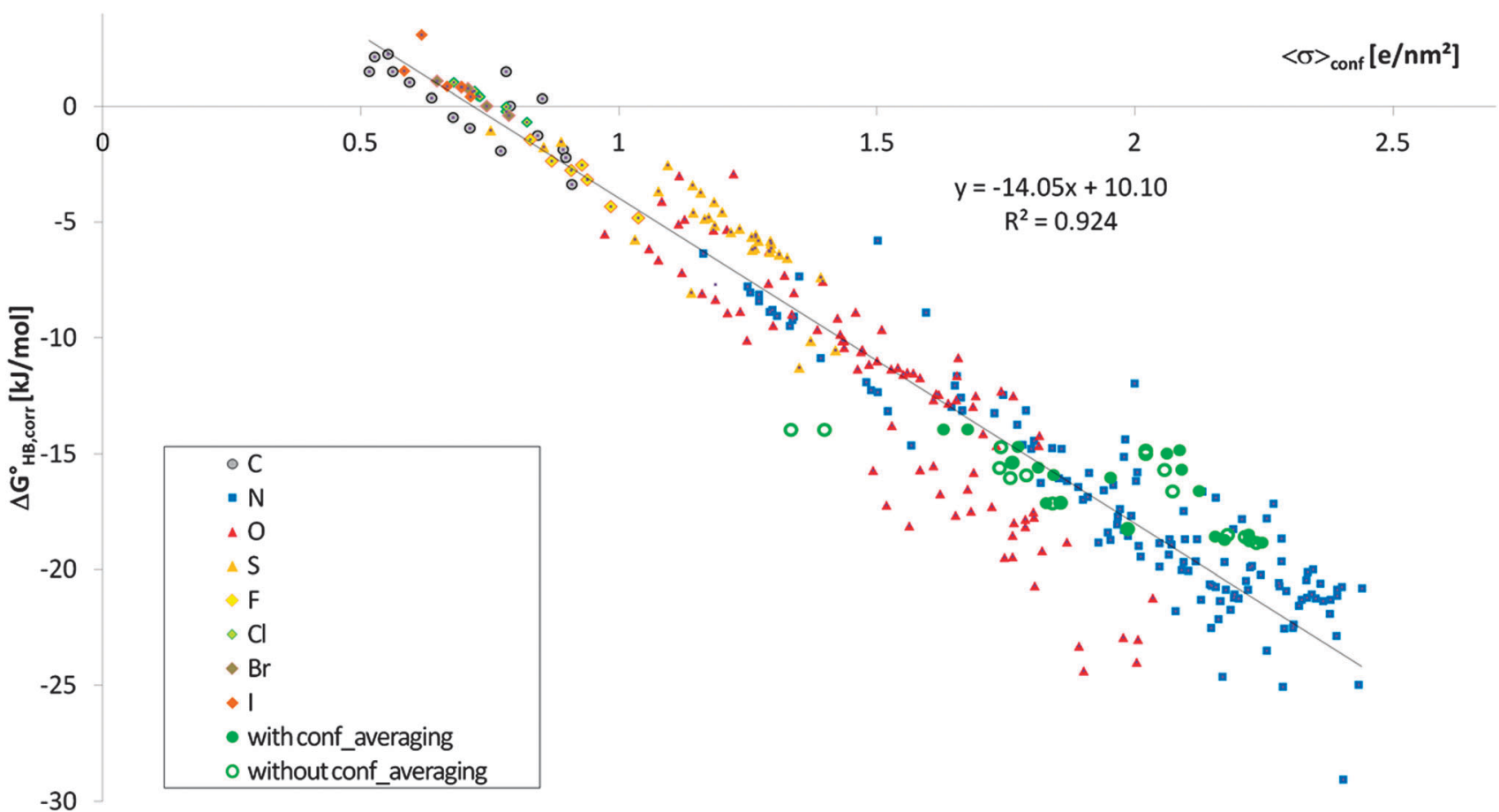

Fig. 6 Experimental $\mathrm{HB}$ free energies, $\Delta \mathrm{G}_{\mathrm{HB}}^{\mathrm{O}}$, corr, of the $307 \mathrm{HBA}$ molecules $(289+18)$ corrected for the conformationally averaged $\mathrm{HB}$ partition function plotted vs.

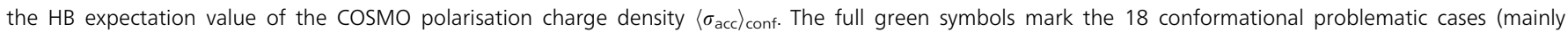
benzylamines). For comparison, open green symbols mark these compounds without conformational averaging.

densities $\sigma$, established for an unprecedented chemically diverse sample, can be used for the prediction of HB enthalpies and free energies of compounds for which experimental data are not available or even not experimentally accessible, e.g. in the case of multiple competing $\mathrm{HB}$ acceptors.

From a theoretical point of view the results present for the first time a quantitative model for the HB entropy in solution. This model demonstrates that entropy contributions arising from the directional flexibility are of comparable importance as the entropy loss which arises from the vibrational restriction of the HB length, which increases proportionally to the $\mathrm{HB}$ strength. This quantitative insight into the different contributions of the HB entropy can be useful for improvements of the HB expressions in solvation models as COSMO-RS, but also for force field improvements.

\section{Appendix 1: construction of the HB partition function}

If we assume that every surface segment $\nu$ with surface area $a_{\nu}$ and polarisation charge density $\sigma_{\nu}$ can form a hydrogen bond of enthalpy

$$
h_{\mathrm{hb}}^{\nu}=c_{\mathrm{hb}} \sigma_{\mathrm{don}} \sigma_{\nu}
$$

with a hydrogen bond donor of polarisation charge density $\sigma_{\mathrm{don}}$, then the relative partition function of hydrogen bonding compared to a reference is

$$
\Omega_{\mathrm{hb}, \mathrm{conf}}^{\alpha}=a_{\mathrm{hb}}{ }^{-1} \sum_{i} w_{i}^{\alpha} \sum_{\nu \in \alpha} a_{\nu} \exp \left\{\frac{-c_{\mathrm{hb}} \sigma_{\mathrm{don}}\left(\sigma_{\nu}-\sigma_{\mathrm{ref}}\right)}{R T}\right\}
$$

where the $\nu$ summation is over all surface segments of a molecule $\alpha$, and $\sigma_{\text {ref }}$ can be either the maximum value of $\sigma_{\text {acc }}$ on the acceptor molecule, or its expectation value according to eqn (A3). The $i$ summation is over the conformations of molecule $\alpha$, where $w_{i}^{\alpha}$ is the relative conformational population of conformation $i$. In the case of just one conformation it is unity. $a_{\mathrm{hb}}$ is the effective hydrogen bond contact area, which has a value of $4.57 \AA$ in the parameterisation used here.

In our case of hydrogen bonds with a fixed donor $(p \mathrm{FP})$ in a fixed solvent $\left(\mathrm{CCl}_{4}\right)$ the value of $c_{\mathrm{hb}} \sigma_{\mathrm{don}}$ can be taken from the slope of the HB enthalpy with respect to $\sigma_{\text {acc }}$, i.e. from the slope of the regression line in Fig. 1.

The expectation value of the hydrogen bond $\sigma_{\text {acc }}$ arising from the multiple directional choices of the $\mathrm{HB}$ and potentially from multiple HB sites on the acceptor surface consequently is given as

$$
\begin{aligned}
& \left\langle\sigma_{\mathrm{acc}}\right\rangle_{\mathrm{conf}} \\
& =a_{\mathrm{hb}}{ }^{-1} \sum_{i} w_{i}^{\alpha} \sum_{\nu \in \alpha} \sigma_{\nu} a_{\nu} \exp \left\{\frac{-c_{\mathrm{hb}} \sigma_{\mathrm{don}}\left(\sigma_{\nu}-\sigma_{\mathrm{ref}}\right)}{R T}\right\} / \Omega_{\mathrm{hb}, \mathrm{conf}}^{\alpha}
\end{aligned}
$$

\section{References}

1 R. W. Taft, D. Gurka, L. Joris, P. v. R. Schleyer and J. W. Rakshys, J. Am. Chem. Soc., 1969, 91, 4801-4808.

2 M. H. Abraham, P. P. Duce, D. V. Prior, D. G. Barratt, J. J. Morris and P. J. Taylor, J. Chem. Soc., Perkin Trans. 2, 1989, 1355-1375. 
3 M. H. Abraham, P. L. Grellier, D. V. Prior, J. J. Morris and P. J. Taylor, J. Chem. Soc., Perkin Trans. 2, 1990, 521-529.

4 M. H. Abraham, J. Phys. Org. Chem., 1993, 6, 660-684.

5 O. A. Raevsky, J. Phys. Org. Chem., 1997, 10, 405-413.

6 O. A. Raevsky and K.-J. Schaper, Eur. J. Med. Chem., 1998, 33, 799-807.

7 M. H. Abraham and J. A. Platts, J. Org. Chem., 2001, 66, 3484-3491.

8 C. Laurence, K. A. Brameld, J. Graton, J.-Y. Le Questel and E. Renault, J. Med. Chem., 2009, 52, 4073-4086.

9 C. Laurence and M. Berthelot, Perspect. Drug Discovery Des., 2000, 18, 39-60.

10 C. Laurence, J. Graton, M. Berthelot, F. Besseau, J. Y. Le Questel, M. Lucon, C. Ouvrard, A. Planchat and E. Renault, J. Org. Chem., 2010, 75, 4105-4123.

11 J. A. Platts, D. Butina, M. H. Abraham and A. Hersey, J. Chem. Inf. Comput. Sci., 1999, 39, 835.

12 P. W. Kenny, J. Chem. Soc., Perkin Trans. 2, 1994, 199-202.

13 J. Schwöbel, R.-U. Ebert, R. Kühne and G. Schüürmann, J. Comput. Chem., 2009, 30, 1454.

14 J. Schwöbel, R.-U. Ebert, R. Kühne and G. Schüürmann, J. Chem. Inf. Model., 2009, 49, 956.

15 J. Schwöbel, R.-U. Ebert, R. Kühne and G. Schüürmann, J. Phys. Chem. A, 2009, 113, 10104.

16 J. Schwöbel, R.-U. Ebert, R. Kühne and G. Schüürmann, J. Phys. Org. Chem., 2011, 24, 1072-1080.

17 A. Klamt, Chemosphere, 1993, 32, 171-726.

18 A. Klamt, J. Reinisch, F. Eckert, A. Hellweg and M. Diedenhofen, Phys. Chem. Chem. Phys., 2012, 14, 955-963.

19 A. Klamt and G. Schüürmann, J. Chem. Soc., Perkin Trans. 2, 1993, 799-805.

20 A. Klamt, J. Phys. Chem., 1995, 99, 2224-2235.
21 A. Klamt, V. Jonas, T. Bürger and J. C. W. Lohrenz, J. Phys. Chem. A, 1998, 102, 5074-5085.

22 A. Klamt, COSMO-RS: From quantum Chemistry to Fluid Phase Thermodynamics and Drug Design, Elsevier, Amsterdam, 2005.

23 J. Graton, M. Berthelot and C. Laurence, J. Chem. Soc., Perkin Trans. 2, 2001, 2130-2135.

24 TURBOMOLE V6.3 2011, a development of University of Karlsruhe and Forschungszentrum Karlsruhe $\mathrm{GmbH}$, 1989-2007, TURBOMOLE GmbH, since 2007, http:/www. turbomole.com.

25 A. D. Becke, Phys. Rev. A: At., Mol., Opt. Phys., 1988, 38, 3098-3100.

26 J. P. Perdew, Phys. Rev. B: Condens. Matter Mater. Phys., 1986, 33, 8822-8824.

27 J. P. Perdew, Phys. Rev. B: Condens. Matter Mater. Phys., 1986, 33, 8822-8824.

28 A. Schäfer, C. Huber and R. Ahlrichs, J. Chem. Phys., 1994, 100, 5829-5835.

29 A. Schäfer, H. Horn and R. Ahlrichs, J. Chem. Phys., 1992, 97, 2571-2577.

30 D. Rappoport and F. Furche, J. Chem. Phys., 2010, 133, 134105.

31 A. Klamt, F. Eckert and M. Diedenhofen, J. Phys. Chem. B, 2009, 113, 4508.

32 COSMOtherm, Version C2.1, Release 01.12, COSMOlogic $\mathrm{GmbH} \&$ Co. KG, Leverkusen, Germany, 2011.

33 J. Graton, M. Berthelot, F. Besseau and C. Laurence, J. Org. Chem., 2005, 70, 7892-7901.

34 W. Adcock, J. Graton, C. Laurence, M. Lucon and N. Trout, J. Phys. Org. Chem., 2005, 18, 227-234.

35 D. Reyntlens-Van Damme and T. Zeegers-Huyskens, J. Phys. Chem., 1980, 84, 282-285.

36 A. Klamt, V. Jonas, T. Bürger and J. C. W. Lohrenz, J. Phys. Chem. A, 1998, 102, 5074-5085. 